support systems in neonatology, further exploration to overcome guideline variations is necessary. Uniformity in neonatal dosage recommendations of antibiotics can also serve as a basis for developing a set of neonatal defined daily doses (nDDDs), a valuable tool for analysis of drug consumption.

309

POSITIVE IMPACT ON SURVIVAL FOR CHILDREN WITH AIDS IN BRAZIL: COMBINED ANALYSIS OF TWO NATIONAL STUDIES, 19831998 AND 1999-2002

L.H. Matida ${ }^{1}$, A.N. Ramos Junior ${ }^{2}$, J. Heukelbach ${ }^{2}$, A. Sanudo ${ }^{3}$, R.C.M. Succi ${ }^{4}$, H.H.S. Marques ${ }^{3}$, M. Della Negra ${ }^{5}$, N. Hearst ${ }^{6}$, Brazilian Study Group of Survival in Children with AIDS

${ }^{1}$ State Program of STD/AIDS, Sao Paulo, ${ }^{2}$ Federal University of Ceara, Fortaleza, ${ }^{3}$ University of Sao Paulo, ${ }^{4}$ Federal University of Sao Paulo, ${ }^{5}$ Emilio Ribas Institute, Sao Paulo, Brazil, ' $U$ niversity of California - San Francisco, San Francisco, CA, USA

Background: Brazil is known for its AIDS control program, including universal access to prevention, diagnosis, and treatment. Survival data is one indicator for program monitoring and evaluation. However, there are no systematic data on survival of children with AIDS.

Methods: This analysis is based on 2 retrospective cohort studies of children under age 13 diagnosed in 1983-1998 and 1999-2002 ( $\mathrm{N}=914$ and 920, respectively). Both were randomly sampled from all reported cases in the country and employed similar methods. We calculated probability of survival to 60 months for children diagnosed with AIDS due to vertical transmission.

Results: In the first study (1983-1998), overall survival to 60 months was $52.8 \%(95 \% \mathrm{Cl}: 41.9 \%$ $60.8 \%)$. In the second study (1999-2002), overall survival increased to $86.5 \%(83.0 \%-89.3 \%)$. During the entire period, the probability of survival to 60 months increased by 4.5 -fold, rising from about $20 \%$ to $90 \%$ (see figure; $p<.001$ by log-rank test). Among the 16 cases diagnosed 1983-1987, survival was $19.7 \%(11.2 \%-30.0 \%)$. For the 265 cases in 19881992 , it was $27.3 \%$ (22.9\%-31.9\%). For 1993-1994, survival was $39.7 \%(\mathrm{~N}=196 ; 33.4 \%-45.9 \%)$. For $1995-1996$, it was $59.5 \%(\mathrm{~N}=221 ; 52.8 \%-65.6 \%)$. For $1997-1998$, it was $68.2 \% \quad(\mathrm{~N}=216 ; 61.6 \%$ $73.9 \%)$. For $1999-2000$, it was $86.5 \% \quad(\mathrm{~N}=470$;
$83.0 \%-89.3 \%$ ); and for $2001-2002$, it was $90.2 \%$ $(\mathrm{N}=450 ; 87.0 \%-92.7 \%)$.

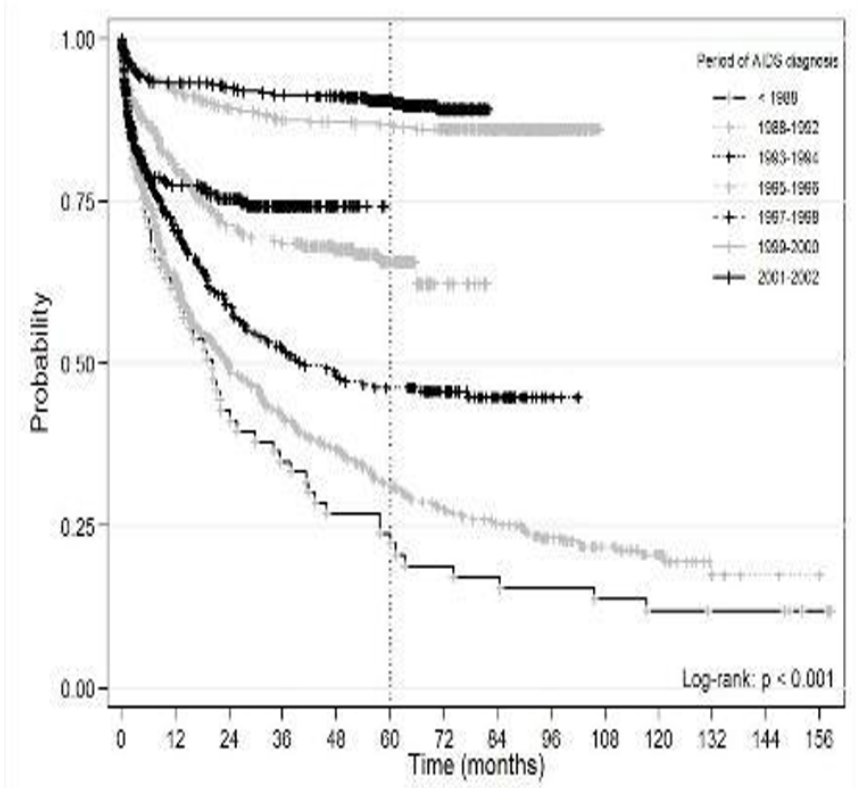

[Survival Curve - Children with AIDS, Brazil]

Conclusions: These results demonstrate the impact of Brazilian policies for children with AIDS. But this achievement creates new challenges related to providing longitudinal comprehensive care and maintaining high quality as well as quantity of survival.

310

TOWARDS ELIMINATION OF VERTICAL TRANSMISSION OF HIV IN SÃO PAULO STATE, BRAZIL - ANALYSIS OF THE PERIOD 1984-2008

L.H. Matida ${ }^{1}$, A.N. Ramos Junior ${ }^{2}$, M.H. Silva ${ }^{1}$, C.S.B. Domingues ${ }^{1}$, A. Tayra ${ }^{1}$, M.C. Gianna ${ }^{1}$, N. Hearst ${ }^{3}$, Study Group of Vertical Transmission HIV/Syphilis, Sao Paulo, Brazil

${ }^{1}$ State Program of STD/AIDS Sao Paulo, Brazil, ${ }^{2}$ Federal University of Ceara, Sao Paulo, Brazil, ${ }^{3}$ University of California - San Francisco, San Francisco, CA, USA

Background: São Paulo State has been a pioneer in adopting measures for prevention of mother-tochild transmission (MTCT) of HIV since the mid1990s. Consequently, HIV infection in children has steadily diminished. Reported AIDS cases in children under five is a useful proxy for MTCT.

Methods: We studied AIDS incidence rates in children under five in São Paulo (Brazil southeast) 
Poster Presentation Abstracts

in period 1984-2008. Cases were tabulated using government reporting system (SINAN), with denominators from Brazilian Institute of Geography and Statistics based on census and inter-census estimates.

Results: During 1984-1996, there was an increase in incidence rates from 0.03 to 12.77 per 100,000 population, an increase of 425 -fold. After implementation of HAART universal access (1996 to present), there was a significant reduction in incidence. The incidence rate fell $90.6 \%$, from 12.77 to 1.20 cases per 100,000 . This drop took place mainly after 2000, when routine diagnostic and preventive strategies were implemented for pregnant women and exposed children. The figure plots incidence by year, indicating when various preventive measures were adopted.

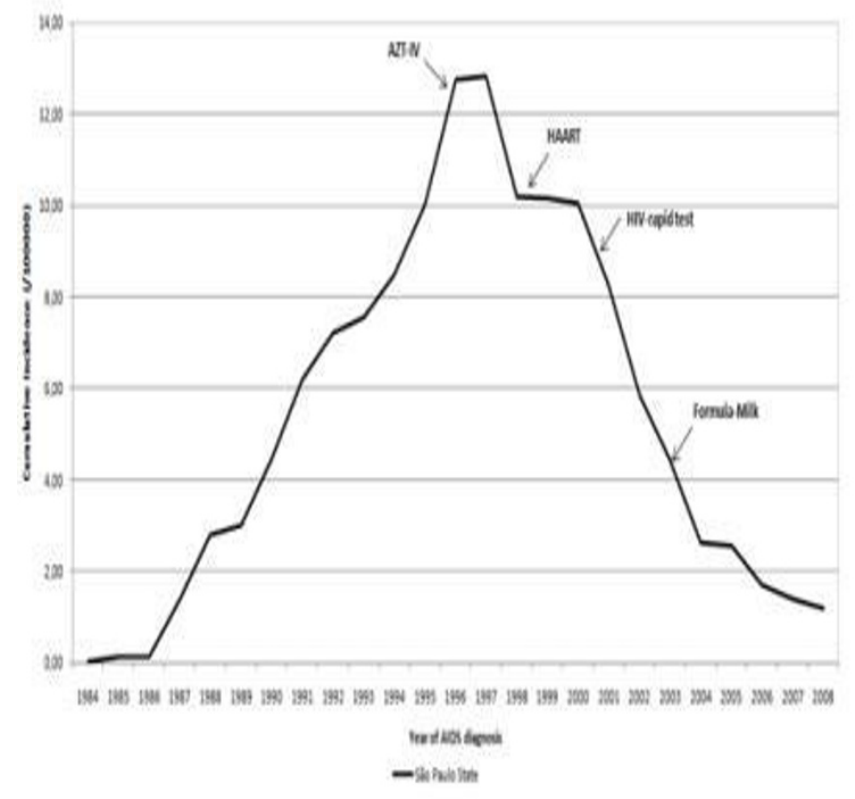

[Vertical Transmission of AIDS, Sao Paulo, Brazil]

Conclusions: AIDS incidence in children is falling in São Paulo State due to adoption of effective control strategies. International goals (i.e., two cases per 100 infected women) for "eliminating" MTCT will certainly be met. Nevertheless, maintaining preventive activities and comprehensive care will remain a challenge in settings with broad social inequality. The 70 cases of AIDS in children under five diagnosed in São Paulo State in 2008 serve as a reminder of this.
311

\section{CLINICAL SIGNIFICANCE OF ROTAVIRUS INFECTION IN NICU: PROSPECTIVE ASSESSMENT OF 60 INFANTS}

M. Spinelli, E. Tridapalli, M.G. Capretti, L. Corvaglia, A. Aceti, G. Faldella

Neonatology and Neonatal Intensive Care Unit - S. Orsola-Malpighi Hospital - University of Bologna, Bologna, Italy

Background and Aims: Although Rotavirus (RV) infection in newborns is usually asymptomatic, outbreaks have been reported, associated with increased incidence of necrotizing enterocolitis (NEC). We conducted a prospective study during the epidemic peak of $\mathrm{RV}$, in order to assess the rate of infection, its clinical importance and the presence of gut inflammation.

Materials and methods: Between February $1^{\text {st }}$ and March 15 ${ }^{\text {th }}, 2009$ all the infants hospitalized for more than 48 hours were enrolled. Faeces were collected daily: the presence of RV was evaluated by immunoassay and the value of Calprotectin by ELISA test.

Results: 60 infants (381 faecal samples) were evaluated: 17 infants (28\%) were RV positive. RV positivity rate was higher in preterm than in term infants (preterm:13/33 vs term:4/27; $p=0.04$ ) and in VLBW (VLBW:9/17 vs non-VLBW:8/43; $p=0.01$ ); no difference in clinical symptoms between RV+ and RV- infants was found. Two infants had blood mucus stools concomitant to the first RV positivity; three RV+ $(17.6 \%)$ and three RV- $(6.9 \%)$ infants had NEC>stagell. No differences in faecal Calprotectin were found among infants non developing NEC (RV+/NEC-: $155 \pm 134$ mg/g vs RV-/NEC-: 116 \pm 124 ); higher values were detected in infants with NEC, regardless of RV (RV+/NEC+: $287 \pm 174$ vs RV-l NEC+: 283 \pm 207 )

Conclusions: RV infection is frequent in NICU during epidemic peak, and more likely in premature than in term infants, partly because of their prolonged hospitalization. Isolated RV infection does not lead to intestinal inflammation and does not seem to favour NEC. 